\title{
Vibrational Analysis of Peptides, Polypeptides and Proteins

\author{
XII-Fermi Resonance Analysis of the Unperturbed ND Stretching \\ Fundamental in Polypeptides
}

\author{
S. Krimm and Anil M. Dwivedi \\ Biophysics Research Division, The University of Michigan, Ann Arbor, Michigan 48109, USA
}

\begin{abstract}
We have obtained the spectrum of $\beta$-poly(L-alanine-ND), and find three bands in the ND stretching region. By comparison with Fermi resonance analyses of deuterated derivatives of polyglycine $I$ as well as with $\alpha-p o l y(L-a l a n i n e-N D)$, we conclude that a three-level Fermi resonance is involved in the $\beta$ polypeptide, and that the unperturbed ND stretch fundamental occurs near $2428 \mathrm{~cm}^{-1}$.
\end{abstract}

\section{INTRODUCTION}

The presence in the infrared spectra of polypeptides of two bands involving the NH stretch mode has long been known. ${ }^{1}$ These bands, a strong one near $3300 \mathrm{~cm}^{-1}$ (referred to as amide A) and its weak counterpart near $3100 \mathrm{~cm}^{-1}$ (referred to as amide $B$ ), have been associated with NH stretch because of their disappearance on N-deuteration. ${ }^{2}$ The suggestion ${ }^{3}$ that these two bands arise from Fermi resonance between the NH stretch fundamental and the first overtone of the (primarily) NH inplane bend mode near $1550 \mathrm{~cm}^{-1}$ (amide II) was supported by studies on an $\mathrm{N}^{15}$ substituted molecule. ${ }^{4}$ Subsequent work ${ }^{5}$ provided a quantitative treatment for this interaction and for the observed intensity ratios of the bands. An analysis of these bands in polyglycine $\mathrm{I}^{6}$, however, showed that from symmetry considerations, the first overtone of an amide II mode could not be involved, and that the level interacting with the $\mathrm{NH}$ stretch fundamental was most probably a combination band between an observed amide II mode (at $1517 \mathrm{~cm}^{-1}$ ) and a hitherto unobserved and unpredicted amide II mode (which was computed to be at about $1588 \mathrm{~cm}^{-1}$ ). This proposal received strong support from a detailed normal mode calculation on polyglycine $\mathrm{I}^{7}$, in which it was shown that a band is predicted near $1588 \mathrm{~cm}^{-1}$ when transition dipole coupling interactions ${ }^{8,9}$ are incorporated in the force field. Similar results were obtained for other $\beta$ polypeptides. ${ }^{7}$ In the case of $\alpha$-helical poly(L-alanine), however, it was shown $^{7,10}$ that an interaction between NH stretch and a first overtone of amide II could indeed be the origin of the amide $\mathrm{A}$ and $\mathrm{B}$ bands.

The analysis of the $\mathrm{NH}$ stretching region of the spectra of polypeptides is thus relatively secure. A much less systematic study has been made, however, of the ND stretching region. Such an analysis is of importance in terms of developing reliable force fields for these molecules. In connection with a calculation of the normal modes of isotopically substituted polyglycine I molecules $^{11}$ and of $\beta$-poly(L-alanine-ND), ${ }^{12}$ we have undertaken a more detailed Fermi resonance analysis of the ND stretching region. We present these results here.

\section{EXPERIMENTAL}

Data on the isotopic polyglycine I species were obtained from published band frequencies and qualitative intensities. ${ }^{13}$ Since no spectra were published, nor quantitative intensities given, our analysis is based on assumed intensity ratios (discussed below).

The spectra of $\beta$-poly(L-alanine-ND) were obtained in our laboratory. ${ }^{12}$ Deuteration (which was never complete, although repeated treatments were used) was performed by dissolving the polypeptide in deuterated trifluoroacetic acid (TFA-D) or by heating in $\mathrm{D}_{2} \mathrm{O}$. The room and liquid $\mathrm{N}_{2}$ temperature spectral contours in the ND stretching region of $\mathrm{KBr}$ pellets of a $\mathrm{D}_{2} \mathrm{O}$ treated polypeptide are shown in Fig. 1.

\section{DISCUSSION}

\section{Polyglycine I}

An analysis of the $\mathrm{NH}$ stretching region of polyglycine I has already been given. ${ }^{7}$ We review this here, in the light of a new normal mode calculation, ${ }^{11}$ and to provide a background to the subsequent analysis of the ND stretching region.

As before, ${ }^{5,7}$ we assume a two-level Fermi resonance, that is, the interaction of the NH stretch fundamental (which for the antiparallel chain rippled sheet structure has $B_{u}$ symmetry ${ }^{7}$ ) with a combination band involving two amide II modes. Under these circumstances, the observed (perturbed) frequencies, $v_{\mathrm{A}, \mathrm{B}}$, are given in terms of the unperturbed frequencies, $v_{\mathrm{A}, \mathrm{B}}, \mathrm{by}^{5}$

$$
\begin{aligned}
& v_{\mathrm{A}}=\frac{1}{2}\left[\left(v_{\mathrm{A}}^{0}+v_{\mathrm{B}}^{0}\right)+\mathrm{s}\right] \\
& v_{\mathrm{B}}=\frac{1}{2}\left[\left(v_{\mathrm{A}}^{0}+v_{\mathrm{B}}^{0}\right)-\mathrm{s}\right]
\end{aligned}
$$



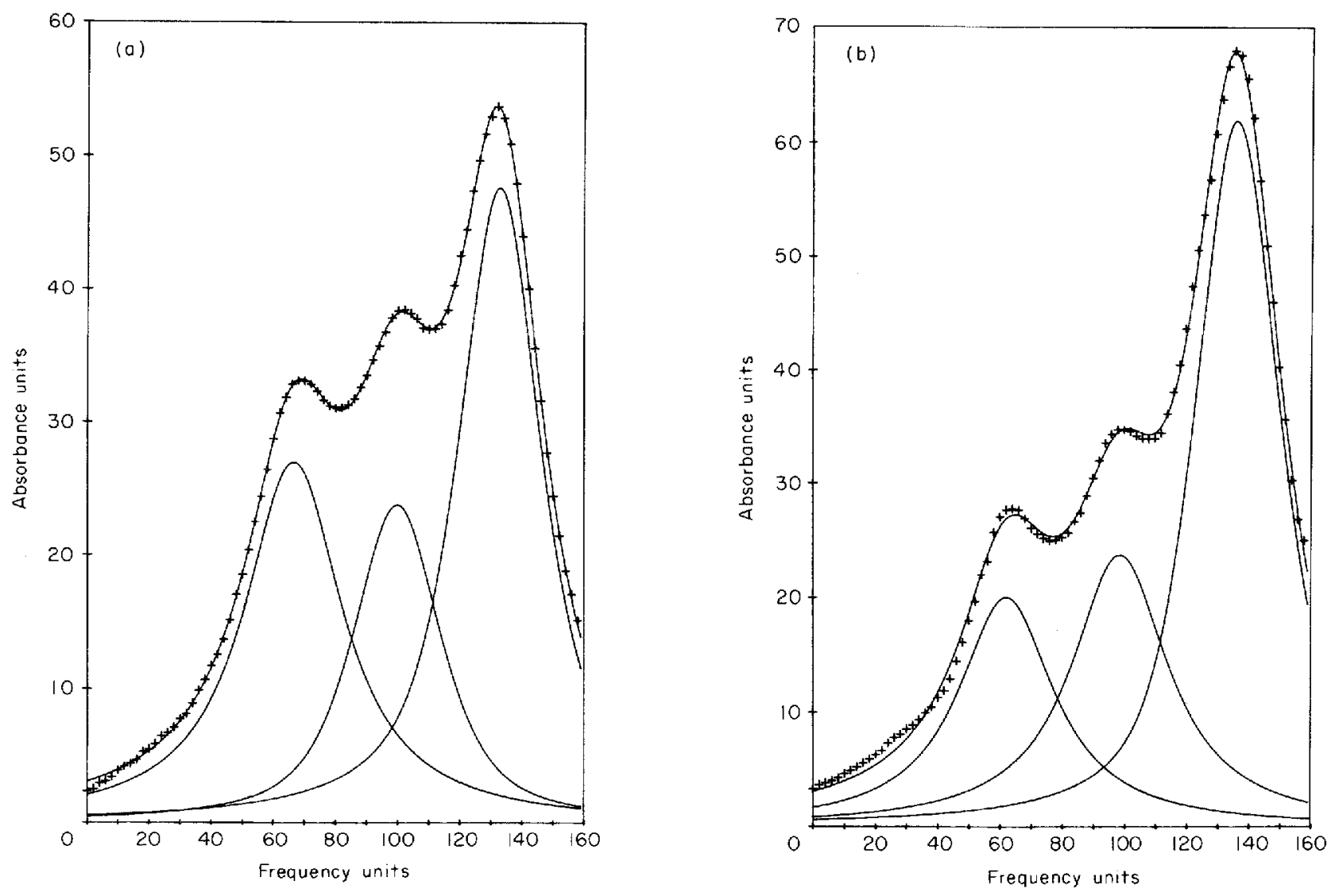

Figure 1. Spectral contours in ND stretching region of $\beta$-poly(L-alanine-ND) prepared by exchange in $\mathrm{D}_{2} \mathrm{O}$. Crosses are observed absorbances (on an arbitrary scale). The curve through them is the sum of indicated component bands. Frequency scale: $0.00=$ $2548 \mathrm{~cm}^{-1}, 160.00=2388 \mathrm{~cm}^{-1}$. (a) Spectrum at room temperature, (b) spectrum at liquid $\mathrm{N}_{2}$ temperature.

where $s=v_{A}-\nu_{B}$. It can also be shown ${ }^{5}$ that

$$
I_{\mathrm{R}} \equiv I_{\mathrm{B}} / I_{\mathrm{A}}=\frac{\mathrm{s}-\delta}{\mathrm{s}+\delta}
$$

where $\delta=v_{\mathrm{A}}^{0}-v_{\mathrm{B}}^{0}$. Thus, a measurement of $\nu_{\mathrm{A}}, \nu_{\mathrm{B}}$, and $I_{\mathrm{R}}$ permits the determination of $\nu_{\mathrm{A}}^{0}$ and $\nu_{\mathrm{B}}^{0}$.

In Table 1, we present the results of such calculations for the various isotopic species of polyglycine I. For $\left(\mathrm{NHCH}_{2} \mathrm{CO}\right)_{n}$, the only other possible combination besides $A_{u}+B_{g}$ is $\underline{1515}\left(A_{g}\right)+1572\left(B_{u}\right)=3087\left(B_{u}\right)$ (the underlined frequency is an observed band), but this is a poorer match to the observed $\nu_{\mathrm{B}}^{0}$ of $3108 \mathrm{~cm}^{-1}$ than the combination given. For $\left(\mathrm{NHCD}_{2} \mathrm{CO}\right)_{n}$, no quantitative intensity data were given, ${ }^{13}$ only the designations $\mathrm{s}$ (strong) and $\mathrm{m}$ (medium) for $v_{\mathrm{A}}$ and $v_{\mathrm{B}}$, respectively. Since these were the same designations as those given for $\left(\mathrm{NHCH}_{2} \mathrm{CO}\right)_{n},{ }^{13}$ we have used the same nominal value for $I_{\mathrm{R}}$, recognizing that this may not be correct. In fact, there is no reason to think that $v_{\mathrm{A}}^{0}$ should be different for these two molecules, and a value of $I_{\mathrm{R}}=$ 0.12 would give $\nu_{\mathrm{A}}^{0}=3271$ (and $\nu_{\mathrm{B}}^{0}=3081$ ). (A common problem with values of $I_{\mathrm{R}}$ given in the literature heretofore is that an accurate quantitative value for this ratio is not available, because resolution of band contours has not been done. We estimate, however, that this introduces an error of no more than about $\pm 3 \mathrm{~cm}^{-1}$ in most computed values of $\nu_{A}^{0}$ and $\nu_{B}^{0}$.) The most important point, however, is that $\nu_{\mathrm{B}}^{0}$ is significantly lower here than for $\left(\mathrm{NHCH}_{2} \mathrm{CO}\right)_{n}$, and this is well accounted for by the somewhat lower frequency amide II bands of the C-deuterated molecule. For $\left(\mathrm{N}^{15} \mathrm{HCH}_{2} \mathrm{CO}\right)_{n}$, we have again used the same value of $I_{\mathrm{R}}$, and we see that

Table 1. Fundamental NH and ND stretching frequencies, and combinations, for polyglycine $\mathrm{I}$.

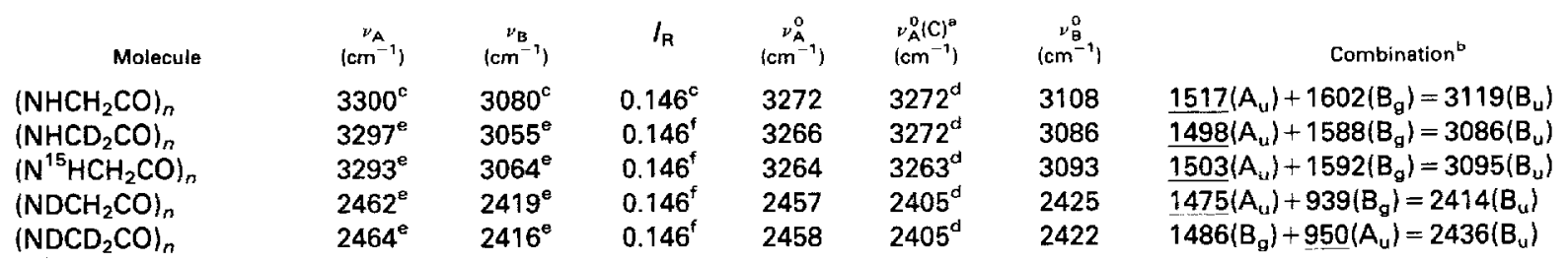

${ }^{\text {a }}$ Calculated frequency, from Ref. 11.

b Underlined frequencies are observed infrared bands; others are calculated values (Ref. 11).

${ }^{c}$ From Ref. 7.

d Force constant adjusted to give $v_{\mathrm{A}}^{\mathrm{o}}(\mathrm{NH})=3272 \mathrm{~cm}^{-1}$ (Ref. 11).

${ }^{\theta}$ From Ref. 13.

${ }^{f}$ Assumed value (see text). 
both the expected drop in the NH stretch fundamental and the intermediate value of the combination band are well explained. These results confirm the original analysis $^{7}$ that the origin of the combination band is in $A_{u}+B_{g}$ amide II modes rather than $A_{g}+B_{u}$ modes; in fact, calculations ${ }^{11}$ show that the latter combination has a smaller value for $\left(\mathrm{N}^{15} \mathrm{HCH}_{2} \mathrm{CO}\right)_{n}$ than for $\left(\mathrm{NHCD}_{2} \mathrm{CO}\right)_{n}$, in disagreement with observation. In all of the above cases, the amide modes consist mainly of $\mathrm{NH}$ in-plane bend with some $\mathrm{CN}$ stretch and $\mathrm{C}^{\alpha} \mathrm{C}$ stretch.

For the analysis of the ND stretching region, we have again had to use a nominal value of $I_{\mathrm{R}}$ because of the absence of quantitative information. ${ }^{13}$ However, reasonable changes in $I_{\mathrm{R}}$ result in only $\pm 2 \mathrm{~cm}^{-1}$ changes in $\nu_{\mathrm{A}}^{0}$ and $\nu_{\mathrm{B}}^{0}$. For $\left(\mathrm{NDCH}_{2} \mathrm{CO}\right)_{n}$, we see first that the calculated value for $v_{A}^{0}$ is about $50 \mathrm{~cm}^{-1}$ below the observed value. This is probably a result of the real difference in anharmonicities between the NH and ND stretch modes, which is not accounted for in our harmonic calculation (the difference between observed and calculated ND stretch frequencies is in the right direction for this effect). Second, the most reasonable combination band is again $A_{u}+B_{g}$, with the former involving mainly $\mathrm{C}^{\alpha} \mathrm{C}$ stretch and $\mathrm{CN}$ stretch, and the latter having a significant ND in-plane bend component. ${ }^{11}$ In this respect, there is a certain analogy with the combination bands for the NH cases. (The only other possible combination of this kind is ${ }^{11} 1485\left(A_{\mathrm{g}}\right)+932\left(\mathrm{~B}_{\mathrm{u}}\right)=2417\left(\mathrm{~B}_{\mathrm{u}}\right)$, which cannot be completely excluded.) For $\left(\mathrm{NDCD}_{2} \mathrm{CO}\right)_{n}$, no combination of the above form (i.e., an $A_{u}$ or $A_{g}$ mode near $1480 \mathrm{~cm}^{-1}$ ) is possible if we wish the band near $900 \mathrm{~cm}^{-1}$ to have an ND in-plane bend component: for this molecule, there are no $\mathrm{B}_{\mathrm{g}}$ or $B_{u}$ modes in the $900-1000 \mathrm{~cm}^{-1}$ region that have ND in-plane bend contributions. ${ }^{11}$ The $B_{g}+A_{u}$ combination, however, seems reasonable (as would a comparable $B_{u}+A_{g}$ combination ${ }^{11}$ ).

Thus, the two bands in the ND stretching region of $\mathrm{N}$-deuterated polyglycine I molecules can be assigned, as in the $\mathrm{NH}$ species, to a Fermi resonance doublet in which the strong, high frequency component is predominantly ND stretch and the weaker low frequency component is a combination band involving a mode with $\mathrm{C}^{\alpha} \mathrm{C}$ and $\mathrm{CN}$ stretch near $1480 \mathrm{~cm}^{-1}$ and a mode with significant ND in-plane bend near $950 \mathrm{~cm}^{-1}$. The necessary presence of the latter component seems reasonable in terms of the localized nature of the ND stretch mode.

\section{Poly(L-alanine)}

An examination of the ND stretching region of $\beta-$ poly(L-alanine-ND) (Fig. 1) shows two significant differences when compared with the spectra of polyglycine I-ND: there are three bands instead of two and the more intense band seems to be the one at lowest frequency. If Fermi resonance accounts for this triplet, then this resonance is presumably not exactly the same as that in polyglycine $I$; for example, a three-level resonance may be involved. Before examining this situation in detail, we will review the analyses for the hydrogenated molecules, both $\alpha$-helical as well as $\beta$.

For $\alpha$-poly(L-alanine), it has been established ${ }^{7,10}$ that a Fermi resonance between NH stretch (A species) and an overtone of amide II ( $E_{1}$ species) can explain the observed amide A and B bands. This is reproduced in Table 2, for both room temperature and low temperature spectra. ${ }^{10}$ (We note that an overtone of the A species amide II mode, found ${ }^{10,14}$ at $1516 \mathrm{~cm}^{-1}$, cannot account for the observed $v_{\mathrm{B}}^{0}$.) It will be seen, incidentally, that $v_{\mathrm{A}}^{0}$ decreases significantly, by $29 \mathrm{~cm}^{-1}$, when the temperature is lowered, undoubtedly reflecting the significant increase in hydrogen bond strength as the helix contracts axially. For $\beta$-poly(L-alanine), $v_{A}$ and $\nu_{\mathrm{B}}$ are accounted for by a Fermi resonance entirely analogous to that in polyglycine I, involving combinations between amide II modes of similar symmetry (the $\mathrm{NH}$ stretch fundamental for the antiparallel chain

Table 2. Fundamental NH and ND stretching frequencies, and combinations, for $\alpha$ - and $\beta$-poly(L-alanine)

\begin{tabular}{|c|c|c|c|c|c|c|c|c|}
\hline & Molecule & $\left(\mathrm{cm}^{-1}\right)$ & $\left(\mathrm{cm}^{-1}\right)$ & $t_{R}$ & $\left(\mathrm{~cm}^{-1}\right)$ & $\begin{array}{l}\nu_{A}^{0}(C)^{a} \\
\left(\mathrm{~cm}^{-1}\right)\end{array}$ & $\left(\begin{array}{c}\nu_{B}^{0} \\
\left(\mathrm{~cm}^{-1}\right)\end{array}\right.$ & Combination \\
\hline \multirow[t]{2}{*}{$\alpha-\mathrm{NH}:$} & $\mathbf{R} \mathbf{T}^{\mathrm{c}}$ & $3307^{d}$ & $3058^{d}$ & $0.127^{d}$ & 3279 & \multirow[t]{2}{*}{$3279^{\circ}$} & 3086 & $2 \times 1545\left(E_{1}\right)=3090(A)$ \\
\hline & $\mathrm{LT}^{\mathrm{c}}$ & $3286^{d}$ & $3070^{d}$ & $0.200^{\mathrm{d}}$ & 3250 & & 3106 & $2 \times \overline{1553}\left(E_{1}\right)=3106(A)$ \\
\hline \multirow[t]{3}{*}{$\beta-N H:$} & RT & $3280^{f}$ & $3072^{4}$ & $0.223^{f}$ & 3242 & \multirow[t]{3}{*}{$3242^{g}$} & 3109 & $1524\left(B_{1}\right)+1593\left(B_{3}\right)=3117\left(B_{2}\right)$ \\
\hline & RT & $3276^{h}$ & $3070^{\mathrm{h}}$ & $0.146^{h}$ & 3250 & & 3096 & $1538(A)+1555\left(B_{2}\right)=3093\left(B_{2}\right)$ \\
\hline & LT & $3263^{h}$ & $3086^{h}$ & $0.128^{h}$ & 3243 & & 3101 & \\
\hline \multirow[t]{3}{*}{$\alpha-N D:$} & $\mathbf{R T}$ & $2464^{d}$ & $2407^{d}$ & $0.35^{h}$ & 2449 & \multirow[t]{2}{*}{$2410^{\theta}$} & 2422 & $1439\left(E_{1}\right)+974\left(E_{1}\right)=2413(A)$ \\
\hline & LT & $2462^{d}$ & $2403^{d}$ & $0.99^{d}$ & 2433 & & 2432 & $1430(\mathrm{~A})+1000(\mathrm{~A})=2430(\mathrm{~A})$ \\
\hline & & $\left(\mathrm{cm}^{-1}\right)$ & $\begin{array}{c}v_{B} \\
\left(\mathrm{~cm}^{-1}\right)\end{array}$ & $\begin{array}{c}\nu_{c} \\
\left\{\mathrm{~cm}^{-1}\right\}\end{array}$ & $\left(\mathrm{cm}^{-1}\right)$ & $\begin{array}{c}\nu_{\mathrm{A}}^{0}(\mathrm{C}) \\
\left(\mathrm{cm}^{-1}\right)\end{array}$ & & Combinations \\
\hline \multirow[t]{2}{*}{$\beta-N D:$} & RT & 2415 & 2448 & 2482 & 2428 & \multirow[t]{2}{*}{$2381^{g}$} & & $\begin{array}{l}1488\left(B_{3}\right)+\underline{999}\left(B_{1}\right)=2487\left(B_{2}\right) \\
\underline{1488}\left(B_{3}\right)+\underline{942}\left(B_{1}\right)=2430\left(B_{2}\right)\end{array}$ \\
\hline & LT & 2411 & 2449 & 2486 & 2419 & & & \\
\hline \multicolumn{9}{|c|}{ 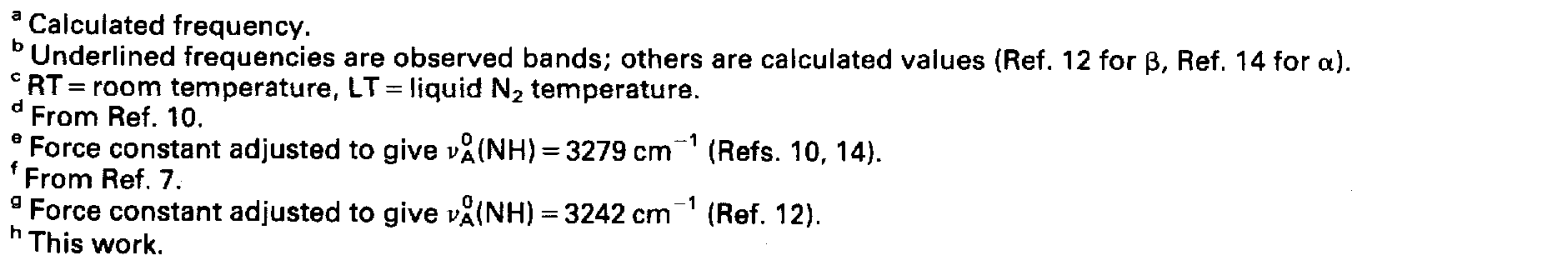 } \\
\hline
\end{tabular}


pleated sheet structure of $\beta$-poly(L-alanine) has $\mathrm{B}_{2}$ symmetry $\left.^{7}\right)$. Our present measurements of $I_{\mathrm{R}}$ would also permit an $\mathrm{A}+\mathrm{B}_{2}$ combination. The much lower value of $v_{\mathrm{A}}^{0}$ reflects the much stronger hydrogen bond in the $\beta(\mathrm{l}(\mathrm{N} \ldots \mathrm{O})=2.73 \AA)$ as compared with the $\alpha(1(\mathrm{~N} \cdots \mathrm{O})=$ $2.83 \AA$ ) polypeptide. The smaller shift on lowering the temperature is undoubtedly a result of the small lateral contraction within the sheet, thus only slightly altering the hydrogen bond strength. Therefore, the analysis of the NH stretch fundamental in poly(L-alanine) follows directly from that of polyglycine $I$.

In the case of $\alpha$-poly(L-alanine-ND), we have reexamined the data ${ }^{10}$ for $I_{\mathrm{R}}$ and feel that the previous room temperature ratio does not adequately account for band overlaps. We believe that a value of $I_{\mathrm{R}}$ closer to half of that originally quoted, ${ }^{10}$ is more realistic. In addition, our new normal mode calculation ${ }^{14}$ indicates that the observed frequency at $\sim 1000 \mathrm{~cm}^{-1}$ should be assigned to an $A$ species rather than an $E_{1}$ species mode, the latter being associated with a band near $974 \mathrm{~cm}^{-1}$. Both of these observations provide a more consistent explanation in terms of an $E_{1}+E_{1}$ combination, although an $\mathrm{A}+\mathrm{A}$ combination can perhaps not be excluded. The situation is complicated by the fact that a weak third band, at $\sim 2450 \mathrm{~cm}^{-1}$, is seen as a shoulder in the low temperature spectrum, ${ }^{10}$ thus raising the possibility that a three-level resonance may be involved. We have not pursued this point further.

On the basis of the above results, we should have certain general expectations about the ND stretch frequency in $\beta$-poly(L-alanine-ND). First, since the hydrogen bond in $\beta$ is stronger than in $\alpha$, resulting in $v_{\mathrm{A}}^{0}(\mathrm{NH})=3242-3250 \mathrm{~cm}^{-1}$ of $\beta$ being lower than the $v_{\mathrm{A}}^{0}(\mathrm{NH})=3279 \mathrm{~cm}^{-1}$ of $\alpha$, we should expect that $\nu_{\mathrm{A}}^{0}$ (ND) of $\beta$ should be lower than $2449 \mathrm{~cm}^{-1}$ $\left(2441 \mathrm{~cm}^{-1}\right.$ if the earlier value ${ }^{10}$ of $I_{\mathrm{R}}$ were used). Second, following the relatively small downward shift $\left(\sim 7 \mathrm{~cm}^{-1}\right)$ on cooling that occurs for $v_{\mathrm{A}}^{0}(\mathrm{NH})$, we would anticipate a similarly small shift for $v_{\mathrm{A}}^{0}(\mathrm{ND})$, certainly less than the $\sim 16 \mathrm{~cm}^{-1}$ shift found for $\alpha-v_{A}^{o}(N D)$. Third, by analogy with polyglycine I-ND and perhaps $\alpha-$ poly(L-alanine-ND), we should expect that the observed $v_{A}^{0}(\mathrm{ND})$ should be no more than $40-50 \mathrm{~cm}^{-1}$ higher than the calculated value based on using the force constant adjusted for $v_{\mathrm{A}}^{0}(\mathrm{NH})$. It is useful to keep these guidelines in mind as we analyze the ND stretching region of $\beta$-poly (L-alanine-ND).

As can be seen from Fig. 1, there are three clearly defined bands in the ND stretching region of the spectrum of the $\beta$ polypeptide. Furthermore, the more intense band is now at the lowest frequency, in distinction to all of the previous cases. We have resolved the observed band contour, using a previously described computer program, ${ }^{15}$ and find three component bands (labelled $v_{A}, v_{B}$, and $\nu_{C}$ ) at the frequencies given in Table 2 (see Fig. 1 for results of the resolution). It is clear that if all three bands are associated with $\beta$-poly $(\mathrm{L}-$ alanine-ND), then we are probably dealing with a threelevel Fermi resonance situation, which has already been proposed for the amide I-amide II region of solid $N$ methylacetamide. ${ }^{16}$ In distinction to that case, we presumably have a resonance between a fundamental and two combination bands, and we therefore expect the strongest observed band to be most closely related to the fundamental. ${ }^{16}$
We believe that the evidence supports the proposal that the three bands are associated with the $\beta$ structure, although there can be a contribution in this region from another (perhaps unordered) component. First, the residual $\mathrm{NH}$ stretch band for the $\mathrm{D}_{2} \mathrm{O}$-treated sample is at the same frequency $\left(3276 \mathrm{~cm}^{-1}\right)$ as the original $\beta$ band, thus suggesting that the treatment has not produced drastic structural changes. Second, in the sample deuterated with TFA-D the residual NH stretch band is at about $3302 \mathrm{~cm}^{-1}$, suggesting contributions from another structure, but the main change is a downward shift in the high frequency component (from $2482 \mathrm{~cm}^{-1}$ to $2478 \mathrm{~cm}^{-1}$ ) and an increase in its intensity. In fact, as will be seen from Table 3 , this strong broad component shifts downward by about $30 \mathrm{~cm}^{-1}$ at low temperature, far more than the observed $2 \mathrm{~cm}^{-1}$ shift for the $\alpha$-helix (cf. Table 2), but consistent with what might be expected for a disordered structure. Third, the intensity ratios $I\left(\nu_{\mathrm{B}}\right) / I\left(\nu_{\mathrm{A}}\right)$ and $I\left(v_{\mathrm{C}}\right) / I\left(\nu_{\mathrm{A}}\right)$ remain about the same between samples, although the other ratios vary in a manner consistent with a contribution from an unordered component. Thus, for both samples at room temperature $I\left(\nu_{\mathrm{B}}\right) / I\left(\nu_{\mathrm{A}}\right)=0.41 \pm 0.10$, whereas $I\left(\nu_{\mathrm{C}}\right) / I\left(\nu_{\mathrm{A}}\right)$ is 0.73 for the $\mathrm{D}_{2} \mathrm{O}$ sample and 1.21 for the TFA-D sample (this reversăl of relative intensity shows that an independent component must be contributing near $2478 \mathrm{~cm}^{-1}$ in the latter sample). Similarly, at low temperature $I\left(\nu_{\mathrm{C}}\right) / I\left(\nu_{\mathrm{A}}\right)=0.55 \pm 0.13$ for both samples, but $I\left(\nu_{\mathrm{B}}\right) / I\left(\nu_{\mathrm{A}}\right)$ is 0.50 for the $\mathrm{D}_{2} \mathrm{O}$ sample and 1.12 for the TFA-D sample (consistent with an independent contribution near $2449 \mathrm{~cm}^{-1}$ in the latter sample). As we will see below, in addition to the general expectations mentioned above as well as the demonstrated persistence of three bands in this region, we also find two appropriate combination bands that can interact with the ND stretch fundamental.

Table 3. Component bands in ND stretching region of $\beta$ poly(L-alanine-ND)

\begin{tabular}{|c|c|c|c|}
\hline & & $\mathrm{D}_{2} \mathrm{O}^{\mathrm{a}}$ & TFA-D $D^{A}$ \\
\hline \multirow[t]{9}{*}{$R T^{b}$} & $v_{A}\left(\mathrm{~cm}^{-1}\right)$ & 2415 & 2416 \\
\hline & $H W H M^{c}\left(\mathrm{~cm}^{-1}\right)$ & 16 & 17 \\
\hline & Area $^{d}$ & 0.448 & 0.398 \\
\hline & $v_{\mathrm{B}}\left(\mathrm{cm}^{-1}\right)$ & 2448 & 2449 \\
\hline & HWHM $\left(\mathrm{cm}^{-1}\right)$ & 17 & 14 \\
\hline & Area & 0.227 & 0.120 \\
\hline & $v_{\mathrm{C}}\left(\mathrm{cm}^{-1}\right)$ & 2482 & 2478 \\
\hline & $\mathrm{HWHM}\left(\mathrm{cm}^{-1}\right)$ & 19 & 25 \\
\hline & Area & 0.325 & 0.482 \\
\hline \multirow[t]{9}{*}{$\mathrm{LT}^{\mathrm{b}}$} & $\nu_{A}\left(\mathrm{~cm}^{-1}\right)$ & 2411 & 2411 \\
\hline & HWHM $\left(\mathrm{cm}^{-1}\right)$ & 16 & 14 \\
\hline & Area & 0.520 & 0.356 \\
\hline & $v_{B}\left(\mathrm{~cm}^{-1}\right)$ & 2449 & 2449 \\
\hline & HWHM $\left(\mathrm{cm}^{-1}\right)$ & 19 & 26 \\
\hline & Area & 0.262 & 0.400 \\
\hline & $\nu_{\mathrm{C}}\left(\mathrm{cm}^{-1}\right)$ & 2486 & 2492 \\
\hline & HWHM $\left(\mathrm{cm}^{-1}\right)$ & 19 & 20 \\
\hline & Area & 0.218 & 0.244 \\
\hline
\end{tabular}

a Sample deuterated with $\mathrm{D}_{2} \mathrm{O}$ or TFA-D.

${ }^{b} \mathrm{RT}=$ room temperature, $\mathrm{LT}=$ liquid $\mathrm{N}_{2}$ temperature.

c HWHM = half-width at half-maximum.

Area relative to that of the total band. 
If we accept the existence of three bands in the ND stretching region, then the frequency of the unperturbed ND stretch mode can be obtained in a straightforward way if we can determine the contributing combination bands. This is because we always have ${ }^{16} \nu_{A}+\nu_{B}+v_{C}=$ $v_{\mathrm{A}}^{0}+\nu_{\mathrm{B}}^{0}+v_{\mathrm{C}}^{0}$. An examination of possible combinations similar to those for $\beta$-poly $(\mathrm{L}$-alanine $)$ indicates $^{12}$ that, for the room temperature spectrum, the only reasonable ones are (a) $\nu_{\mathrm{B}}^{0}=1464\left(\mathrm{~B}_{1}\right)+1020\left(\mathrm{~B}_{3}\right)=2484\left(\mathrm{~B}_{2}\right)$

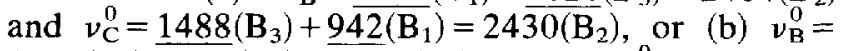
$1488\left(B_{3}\right)+999\left(B_{1}\right)=2487\left(B_{2}\right)$ and $\nu_{C}^{0}=2430\left(B_{2}\right)$. Since $v_{\mathrm{A}}+v_{\mathrm{B}}+\nu_{\mathrm{C}}=7345 \mathrm{~cm}^{-1}$, this gives (a) $\nu_{\mathrm{A}}^{0}=$ $2431 \mathrm{~cm}^{-1}$ or (b) $\nu_{A}^{0}=2428 \mathrm{~cm}^{-1}$. Choice (a) is less reasonable than choice (b), since it would put the fundamental at a higher frequency than a combination band, and the relative intensities suggest that $\nu_{\mathrm{A}}^{0}$ is the lowest frequency. We therefore choose the latter assignments for the contributing combination bands. In the low temperature spectrum, we have observed that the medium intensity band at $1464 \mathrm{~cm}^{-1}$ moves to higher frequency by about $5 \mathrm{~cm}^{-1}$, whereas the 999 and $942 \mathrm{~cm}^{-1}$ bands remain about the same. If we can assume a similar shift in the very weak $1488 \mathrm{~cm}^{-1}$ band (which is hard to detect in the low temperature spectrum), then we find that $v_{\mathrm{A}}^{0}(\mathrm{ND})$ is now $2419 \mathrm{~cm}^{-1}$.

The conclusion that $\nu_{\mathrm{A}}^{0}(\mathrm{ND})$ for room temperature $\beta$-poly(L-alanine-ND) is at about $2428 \mathrm{~cm}^{-1}$ is con- sistent with our expectations. First, it is lower than the $2449 \mathrm{~cm}^{-1}$ of the $\alpha-\mathrm{ND}$ molecule, which is required by the stronger hydrogen bond. Second, it shifts down by only $9 \mathrm{~cm}^{-1}$ on cooling, analogous to the $7 \mathrm{~cm}^{-1}$ shift found for $\beta-v_{A}^{0}(\mathrm{NH})$. Third, the value of $\nu_{\mathrm{A}}^{0}(\mathrm{ND})$ is $47 \mathrm{~cm}^{-1}$ highe than the calculated ND stretch frequency, as expected by analogy with the other polypeptides. Finally, we can see a justification for the three-level Fermi resonance in $\beta$-poly(L-alanine-ND) as compared with the analogous polyglycine I species: whereas in the latter only one combination band is possible in the appropriate region, ${ }^{11}$ for the former we can have two combination bands in the necessary region, ${ }^{12}$ separated by some $55 \mathrm{~cm}^{-1}$. (The expectation of a three-level resonance is, of course, based on the assumption ${ }^{16}$ that otherwise a combination band would exhibit essentially no intensity.)

We therefore propose that in deuterated $\beta$ polypeptides the unperturbed ND stretch frequency is to be found near $2428 \mathrm{~cm}^{-1}$.

\section{Acknowledgement}

This research was supported by National Science Foundation grants PCM-7921652 and DMR-7800753.

This paper is dedicated to the memory of Professor Takehiko Shimanouchi, who played such an important role in opening up the field of vibrational spectroscopic studies of polypeptides and proteins.

\section{REFERENCES}

1. M. Tsuboi, Bull. Chem. Soc. Jpn 22, 255 (1949).

2. C. H. Bamford, A. Elliott and W. E. Hanby, Synthetic Polypeptides, p. 194. Academic Press, New York (1956).

3. R. M. Badger and A. D. E. Pullin, J. Chem. Phys. 22, 1142 (1954).

4. M. Beer, H. B. Kessler and G. B. B. M. Sutherland, J. Chem. Phys. 29, 1097 (1958).

5. T. Miyazawa, J. Mol. Spectrosc. 4, 168 (1960)

6. M. Tsuboi, Biopolym. Symp. 1, 527 (1964).

7. W. H. Moore and S. Krimm, Biopolymers 15, 2439 (1976).

8. S. Krimm and Y. Abe, Proc. Natl Acad. Sci. USA 69, 2788 (1972).

9. W. H. Moore and S. Krimm, Proc. Natl Acad. Sci. USA 72, 4933 (1975).

10. J. F. Rabolt, W. H. Moore and S. Krimm, Macromolecules 10, 1065 (1977).
11. A. M. Dwivedi and S. Krimm, Macromolecules in press.

12. A. M. Dwivedi and S. Krimm, Macromolecules in press

13. S. Suzuki, Y. Iwashita, T. Shimanouchi and M. Tsuboi, Biopolymers 4, 337 (1966).

14. J. Bandekar, A. M. Dwivedi and S. Krimm, unpublished.

15. T. C. Cheam and S. Krimm, J. Polym. Sci., Polym. Phys. Ed. 19, 423 (1981).

16. G. Dellepiane, S. Abbate, P. Bosi and G. Zerbi, J. Chem. Phys. 73, 1040 (1980).

Received 27 May 1981

(C) Heyden \& Son Ltd, 1982 